\title{
Isolation and Characterization of Type I Signal Peptidase of Different Malaria Parasites
}

\author{
Sutikshan Sharma, Arun Pradhan, Virander S. Chauhan, and Renu Tuteja \\ Malaria Group, International Centre for Genetic Engineering and Biotechnology, \\ PO Box 10504, Aruna Asaf Ali Marg, New Delhi 110067, India
}

Received 16 February 2005; revised 12 July 2005; accepted 14 July 2005

\begin{abstract}
Type I signal peptidases are important membrane-bound serine proteases responsible for the cleavage of the signal peptide of the proteins. These enzymes are unique serine proteases that carry out catalysis using a serine/lysine catalytic dyad. In the present study, we report the isolation of type I signal peptidase from the malaria parasites Plasmodium falciparum, Plasmodium knowlesi, and Plasmodium yoelii and some characterization of type I signal peptidase of Plasmodium falciparum. We show that these enzymes are homologous to signal peptidases from various sources and also contain the conserved boxes present in other type I signal peptidases. The type I signal peptidase from $P$ falciparum is an intron-less and a single-copy gene. The results also show that the enzyme from Plasmodium falciparum is subject to self-cleavage and it has been demonstrated to possess type I signal peptidase activity in $E$ coli preprotein processing in vivo by complementation assay. This study will be helpful in understanding one of the important metabolic pathways "the secretory pathway" in the parasite and should make an important contribution in understanding the complex process of protein targeting in the parasite.
\end{abstract}

\section{INTRODUCTION}

Signal peptidases are a widespread family of enzymes which catalyze the hydrolytic cleavage of the $\mathrm{N}$-terminal signal peptide from translocated preproteins thereby releasing secreted proteins from the membrane and allowing them to locate to their final destination $[1,2]$. It has been suggested that the signal peptidase plays a key role in the secretion process by removing the signal peptide and releasing the mature protein. The signal peptidases are unique serine proteases that carry out catalysis using a serine/lysine dyad instead of the prototypical serine/histidine/aspartic acid triad found in most serine proteases. Type I signal peptidase is responsible for the cleavage of signal peptides of a number of secreted proteins in bacteria and serves as a potential target for the development of novel antibacterial agents due to its unique biochemical and physiological properties [2]. So far signal peptidases have been found in bacteria, archaea, fungi,

Correspondence and reprint requests to Renu Tuteja, Malaria Group, International Centre for Genetic Engineering and Biotechnology, PO Box 10504, Aruna Asaf Ali Marg, New Delhi 110067, India, E-mail: renu@icgeb.res.in

This is an open access article distributed under the Creative Commons Attribution License which permits unrestricted use, distribution, and reproduction in any medium, provided the original work is properly cited. plants, and animals. In all the bacteria analyzed so far, type I signal peptidase has been shown to be essential for cell viability $[2,3,4]$. It has been shown that an $E$ coli strain possessing a mutated leader peptidase gene (E coli IT41) has a drastically reduced growth rate [5]. It is interesting to note that the largest number of type I signal peptidases in one single species has thus far been found in the gram-positive eubacterium Bacillus subtilis. Five genes specifying type I signal peptidases are present on the Bacillus subtilis chromosome [6]. The corresponding enzymes denoted by SipS, SipT, SipU, SipV, and SipW, respectively, contain between 168 and 193 amino acids and various studies have shown that these enzymes have different but overlapping substrate specificities $[6,7,8]$. Type I signal peptidases are typically anchored to the membrane by amino-terminal transmembrane segment and have a carboxy-terminal catalytic domain that resides on the outer surface of the cytoplasmic membrane.

It is possible to identify five regions of high-sequence similarity and identity referred to as boxes A-E in type I signal peptidases [9]. Box A corresponds to the transmembrane segment and boxes $\mathrm{B}-\mathrm{E}$ all reside in the carboxy-terminal domain and these contribute to a conserved catalytic type I signal peptidase protein fold [1]. It has been proposed that the Plasmodium trafficking machinery presents various similarities to that of other eukaryotic cells and many of the parasite-secreted polypeptides have a typical eukaryotic signal sequence at the $\mathrm{N}$ terminus $[10,11]$. It has also been demonstrated that 
protein targeting in Plasmodium falciparum is a two-step process mediated by bipartite $N$-terminal presequences that consist of a signal peptide for entry into the secretory pathway and a plant-like transit peptide for subsequent import into the apicoplast [12]. Recently, using the comparative genomic search, a homologue of type I signal peptidase has been identified in Plasmodium falciparum [13]. The evolutionary analysis revealed that the two putative plasmodial type I signal peptidases have three clusters of homologues: bacterial signal peptidases, an Arabidopsis chloroplast thylakoid processing peptidase, and mitochondrial inner membrane peptidases found in eukaryotes, which appear to be the nearest neighbor to the type I plasmodial signal peptidase [13]. The consensus signal peptidase recognition site is Ala-X-Ala, which provides a recognizable cleavage site and alanine is found especially frequently in all the positions of natural cleavage regions [14]. Besides Ala at -1 position, the other residues present at this position could be Ala, Gly, Ser, Cys, or Pro and at -3 position, the other residues could be Ala, Gly, Ser, Cys, Thr, Val, Ile, Leu, or Pro [14]. Recently, the gene encoding type I signal peptidase has been reported from the parasite Leishmania major and it has been shown that it is a significant target of the Leishmania specific immune responses [15]. Molecular and functional characterization of type I signal peptidase from human pathogen Legionella pneumophila has been reported recently and the results show that it is a target for a specific inhibitor of type I signal peptidase [16]. In the present study, we describe the cloning, isolation, and sequence analysis of type I signal peptidase from different malaria parasite species such as Plasmodium falciparum, Plasmodium knowlesi, and Plasmodium yoelii and some functional characterization of type I signal peptidase of Plasmodium falciparum. The type I signal peptidase from Plasmodium falciparum contains the catalytic dyad (serine 175, lysine 274) that is invariable across representative type I signal peptidase proteins with confirmed signal peptidase activity. The results show that the type I signal peptidase from Plasmodium falciparum is intron-less and it may be present only at single copy per haploid genome. Our results also show that all these signal peptidases contain the conserved boxes BE present in signal peptidase from other species and the Plasmodium falciparum signal peptidase is subject to selfcleavage.

\section{MATERIALS AND METHODS}

\section{Isolation of genomic DNA and PCR}

Plasmodium falciparum (strain 3D7) was cultured using human erythrocytes with 5\% haematocrit in RPMI media from Gibco supplemented with 10\% human serum using a protocol described earlier [17]. The genomic DNA from this parasite culture was isolated using standard protocol [18]. The signal peptidase gene was amplified using Plasmodium falciparum genomic DNA as template and oligonucleotide primers. The sequences of all the primers
TABle 1. Primers used in PCR reactions. The nucleotides representing various restriction sites are underlined.

\begin{tabular}{ll}
\hline Name & Nucleotide sequence $\left(5^{\prime} \mathbf{3}^{\prime}\right)$ \\
\hline SPBF1 & GGATCCGCTTATATACAGCTGT TTTAAC \\
SPBR1 & TAAGCTTTCTCCTGAGG TCCGGCTTG \\
YSPF1 & CGGGATCCGAAAAGAACAATATTGGT \\
YSPR1 & GCGTCGACATTAGACATGTACAAAAA \\
KSPF1 & GGGATCCAACATGAACAGAAGTAGC \\
KSPR1 & CCAAGCTTACTCGTTCTGTTACTAAT \\
\hline
\end{tabular}

used for amplification are shown in Table 1. The primers contain restriction sites for cloning. For the amplification of the type I signal peptidases, the forward primer SPBF1 and the reverse primer SPBR1 were used with genomic DNA as template. The PCR conditions were one cycle of denaturation at $94^{\circ} \mathrm{C}$ for 5 min followed by 35 cycles of denaturation at $94^{\circ} \mathrm{C}$ for $1 \mathrm{~min}$, annealing at $54^{\circ} \mathrm{C}$ for $1 \mathrm{~min}$ and extension at $72^{\circ} \mathrm{C}$ for $2 \mathrm{~min}$ and final extension was carried out at $72^{\circ} \mathrm{C}$ for $10 \mathrm{~min}$. Similarly for $P$ yoelii the primer pair YSPF1 and YSPR1 and for $P$ knowlesi the primer pair KSPF1 and KSPR1 were used with their respective genomic DNA as template. The PCR conditions were the same as described above except some variation in the annealing temperature. The PCR products were analyzed by agarose gel electrophoresis. The PCR products from all the amplifications were gel purified and cloned into pGEM-T vector (Promega) to generate the recombinant clones. The obtained DNA clones were sequenced by dideoxy sequencing reactions. The DNA band from the recombinant clone of $P$ falciparum was excised using Bam HI and HindIII enzymes and gel purified for subcloning into the expression vectors.

\section{Southern blotting}

For Southern blotting the Plasmodium falciparum genomic DNA was digested with BglII and NdeI. The digested DNA was electrophoresed on a $0.8 \%$ agarose gel and transferred to Nylon membrane according to standard protocol [18]. The PCR fragment of type I signal peptidase from Plasmodium falciparum was labeled using Random priming kit (Invitrogen, CA, USA) and $\alpha^{32} \mathrm{P}$ dCTP and was used as a probe for hybridization after purification. The membrane was pre-hybridized in 6 XSSC (SSC is $0.15 \mathrm{M} \mathrm{NaCl}, 0.15 \mathrm{M}$ sodium citrate) and $5 \mathrm{X}$ Denhardts solution for $3 \mathrm{~h}$ at $60^{\circ} \mathrm{C}$. Hybridization was done overnight in the prehybridization buffer containing the labeled probe at $55^{\circ} \mathrm{C}$. The blot was washed twice for $5 \mathrm{~min}$ at $25^{\circ} \mathrm{C}$ with $5 \mathrm{X}$ SSC, $0.1 \%(\mathrm{w} / \mathrm{v}) \mathrm{SDS}$ and then twice at $50^{\circ} \mathrm{C}$ for $15 \mathrm{~min}$ with $2 \mathrm{XSSC}$ and $0.1 \%$ SDS. The bands were visualized by autoradiography.

\section{Isolation of RNA and preparation of CDNA}

Total RNA was isolated from asynchronous $P$ falciparum parasite lysate using RNeasy kit from Qiagen 
(GmbH, Germany). The optional step of DNase treatment was also included to remove the genomic DNA contamination. After checking the quality of RNA, this total RNA was used for the preparation of cDNA using cDNA synthesis kit (Superscript first-strand synthesis system from Invitrogen, Carlsbad, Calif, USA). The $P$ falciparum type I signal peptidase was amplified from this cDNA preparation using the forward primer SPBF1 and reverse primer SPBR1 as described above.

\section{Expression and purification of protein}

The complete open reading frame of Plasmodium falciparum signal peptidase was subcloned into expression vectors pET-28b (Novagen) at BamHI at $5^{\prime}$-end and HindIII at $3^{\prime}$-end. Cloning at these sites includes the amino acids of the vector. Finally during protein translation 31 amino acids (including the 6 Histidines) of the vector at the $N$-terminal and 11 amino acids (including the 6 Histidines) at the $\mathrm{C}$-terminal of the protein are added. The expression clones were transformed into $E$ coli strain BL21(DE3)pLysS. Bacteria were grown in LB medium to $A_{600 \mathrm{~nm}}=0.6$. The expression of recombinant protein was induced by $1 \mathrm{mM}$ IPTG for various time points from $30 \mathrm{~min}$ to $4 \mathrm{~h}$. To purify the recombinant protein, the harvested bacterial cells were subjected to sonication after resuspension in lysis buffer $\left(50 \mathrm{mM} \mathrm{Na}_{2} \mathrm{HPO}_{4}\right.$, $\mathrm{pH} 8.0$, and $300 \mathrm{mM} \mathrm{NaCl}$ ). After centrifugation at $4^{\circ} \mathrm{C}$, the pellet was resuspended in denaturing buffer $(8 \mathrm{M}$ urea in lysis buffer, $1 \%$ triton and protease inhibitor cocktail (Sigma)) and sonicated again. After centrifugation at $4^{\circ} \mathrm{C}$, the supernatant was allowed to bind to $\mathrm{Ni}^{2+}$-NTA-agarose matrix (Qiagen, GmbH, Germany) in binding buffer (denaturing buffer supplemented with $40 \mathrm{mM}$ imidazole). The column was extensively washed with binding buffer supplemented with $100 \mathrm{mM}$ imidazole. The recombinant His-tagged proteins were eluted with $500 \mathrm{mM}$ imidazole in binding buffer. The protein was further dialyzed against the same buffer without any $\mathrm{NaCl}$, imidazole, or urea. The final purified protein was checked for purity on $15 \%$ SDSPAGE.

\section{Western blotting}

For Western blotting, the proteins were separated on SDS-PAGE and electrophoretically transferred to nitrocellulose membrane as described [19]. The membrane was blocked in $2 \%$ nonfat milk in Tris-buffered saline (TBS) and incubated with the primary antibody (PentaHis, Qiagen, GmbH, Germany) for $3 \mathrm{~h}$ at room temperature. The blot was washed and incubated for $1 \mathrm{~h}$ with the appropriate secondary antibody (Sigma) coupled to alkaline phosphatase. The blots were developed using BCIP and NBT (Sigma) according to the manufacturer's instructions. In some cases, the secondary antibodies conjugated to horseradish peroxidase were used and the blots were developed using chemiluminescent substrates (ECL-Western blotting detection reagents, Amersham Biosciences, UK) and X-ray film (Kodak).

\section{Complementation assay}

For the complementation assay, various plasmids were transformed into competent $E$ coli strain IT41 cells [5] and selected on plates containing Luria broth (LBampicillin or kanamycin). The plates were incubated at $30^{\circ} \mathrm{C}$ for $48 \mathrm{~h}$. For control, plasmid pET28b was also transformed into $E$ coli strain IT41 cells. For the complementation assay by growth curve, the transformed cells were grown in LB-containing ampicillin or kanamycin at $30^{\circ} \mathrm{C}$ overnight. The resulting cultures were incubated with shaking at nonpermissive temperature at $42^{\circ} \mathrm{C}$ after 100 -fold dilution into fresh LB-media containing ampicillin and kanamycin mixture. The optical density at $600 \mathrm{~nm}$ was recorded at $30 \mathrm{~min}$ intervals for a period of $4-6 \mathrm{~h}$. The experiment was repeated at least three times and the data were plotted.

\section{RESULTS AND DISCUSSION}

For the isolation of type I signal peptidases from various species, the genes were amplified using genomic DNA of various parasites such as $P$ falciparum, $P$ yoelii, and $P$ knowlesi and the primers shown in Table 1. The size of the amplified product for each species is shown in Figure 1a. The sequencing data revealed that the amplified gene for type I signal peptidase of $P$ falciparum is 1047-base pair (PfSPB, Figure 1a, lane 2) (DDBJ/EMBL/GenBank database accession number AY582351) in length and it codes for a protein of 349 amino acids. The blast analysis of PfSPB against "PlasmoDB" database revealed that this gene is located on chromosome 13 of $P$ falciparum and it contains no introns. The calculated molecular weight for PfSPB protein is $\sim 42 \mathrm{kd}$ and the theoretical $\mathrm{pI}$ is 9.77 . The "PlasmoDB" entry number for PfSPB is PF13_0118 and its expression profile shows that the maximum expression is in the "late" trophozoite stages of $P$ falciparum. The sequence contains all the characteristics of signal peptidase family. To confirm the absence of introns in type I signal peptidase from $P$ falciparum, total RNA from asynchronous parasite cultures was isolated. This total RNA was used for the construction of cDNA as described in materials and methods. Type I signal peptidase was amplified using this CDNA as template and the primers SPBF1 and SPBR1. The results revealed that the size of the amplified product is same as the size of the amplified product from genomic DNA (Figure 1b, lanes 2 and 3). The sequencing data of this cDNA showed that the sequence is also identical to the type I signal peptidase amplified from genomic DNA. These data clearly confirm the absence of the introns in type I signal peptidase from $P$ falciparum.

To examine if type I signal peptidase is present in other plasmodium species, full-length gene for type I signal peptidase was amplified from $P$ yoelii and $P$ knowlesi using genomic DNA as template. The sequencing data of the amplified product for type I signal peptidase of $P$ yoelii (PySP, Figure 1a, lane 3) revealed that the gene 


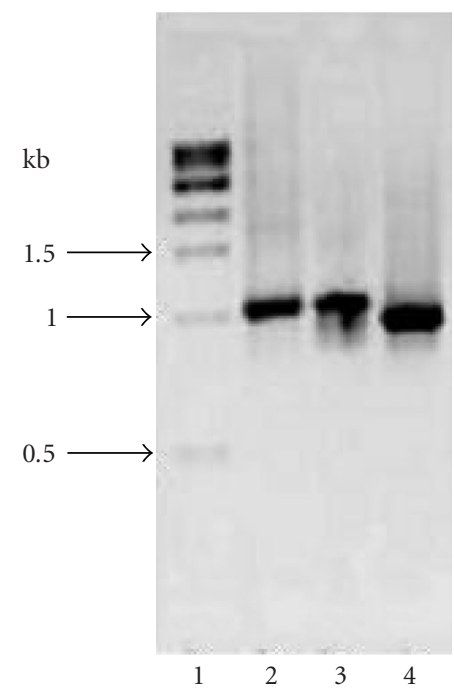

(a)

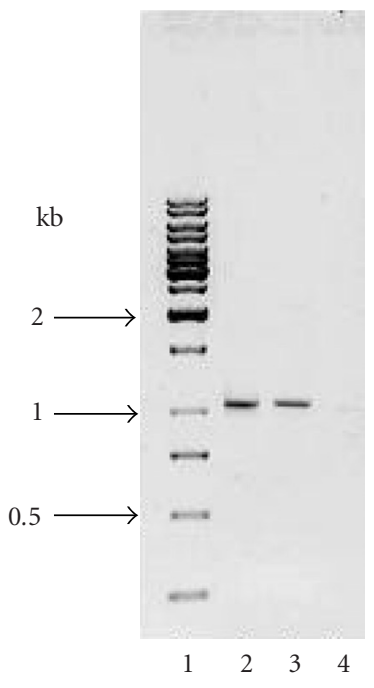

(b)

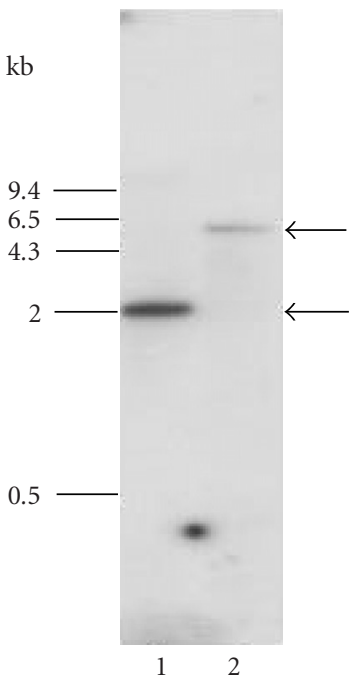

(c)

Figure 1. (a) PCR amplified products of different signal peptidases. The PCR was performed as described in materials and methods and analyzed by agarose gel electrophoresis. Lane 1 is DNA molecular weight marker. Lane 2 is product of $P$ falciparum, lane 3 is $P$ yoelii, and lane 4 is $P$ knowlesi. (b) cDNA analysis of type I signal peptidase of $P$ falciparum. Total RNA isolated from the parasite lysate was used for construction of cDNA and this cDNA was used as template for the amplification of type I signal peptidase as described in materials and methods. Lane 1 is molecular weight marker, lane 2 is PCR product using genomic DNA as template, lane 3 is PCR product using cDNA as template, and lane 4 is PCR reaction without any template. (c) Southern blot analysis of the type I signal peptidase gene of $P$ falciparum. Genomic DNA (20 $\mu$ g per lane) from $P$ falciparum was digested with BglII (lane 1) and NdeI (lane 2) restriction enzymes, separated by electrophoresis on a $0.8 \%$ agarose gel, transferred to a nitrocellulose membrane and hybridized with the type I signal peptidase gene probe of $P$ falciparum. Molecular weight markers are shown on the left side of the autoradiogram and the arrows show the hybridized bands.

is 1038-base pair (DDBJ/EMBL/GenBank database accession number AY787663) (Pyl_02555) contains no introns and it codes for a protein of 346 amino acids (PY07139) with calculated molecular weight of $\sim 41 \mathrm{kd}$ and a theoretical pI of 9.72. The sequencing data of the amplified product for type I signal peptidase of $P$ knowlesi ( $\mathrm{PkSP}$, Figure 1a, lane 4) revealed that the gene is 951 base pair (DDBJ/EMBL/GenBank database accession number AY787662) (Pk_1527d10q1c) contains no introns and it codes for a protein of 317 amino acids with a calculated molecular weight of $\sim 37 \mathrm{kd}$ and a theoretical pI of 10.02 . To estimate the copy number of type I signal peptidase of $P$ falciparum, genomic DNA was isolated by standard methods and analyzed by Southern blotting using various restriction enzymes. Only one band was detected with the restriction enzymes BglII $(\sim 2 \mathrm{~kb})$ and $N d e \mathrm{I}(\sim 5.5 \mathrm{~kb})$ (Figure 1c, lanes 1 and 2). These data suggest that the type I signal peptidase gene in $P$ falciparum may be present only at single copy per haploid genome as reported for other signal peptidases [2].

Using the Mac vector 7.1 program, multiple alignment of the signal peptidases from $P$ falciparum, $P$ yoelii, $P$ knowlesi, human Imp2, Escherichia coli, Saccharomyces cerevisae, Staphylococcus aureus, Streptomyces lividans, and Arabidopsis thaliana was done and $\sim 10-44 \%$ identity and $\sim 6-16 \%$ similarity were observed between the type I $P$ falciparum signal peptidase and its homologue from other species (Figure 2a). The sequence of type I signal peptidase from $P$ falciparum was found to be in very good agreement with virtually all known type I signal peptidases regarding the presence of distinct protein domains. It was observed that the type I signal peptidase from malaria parasites contains the identifiable boxes B$\mathrm{E}$ in the carboxy-terminal catalytic domain, which are also present in all the other known type I signal peptidases. The first conserved region of the sequence is box A, which is part of the hydrophobic segment that helps to anchor the catalytic domain to the membrane. The location of domains B-E of type I signal peptidase of $P$ falciparum is shown in Figure 2b. In $P$ falciparum, box $\mathrm{B}$ contains the residues $173-180$ and the important serine residue at position 175 , which probably serves as the nucleophile in the catalytic reaction. This box also contains a conserved methionine at position 176, which helps in the catalytic mechanism. In $P$ falciparum, box $C$ contains residues 255-264 and in other species it has been shown that the second valine in this box is part of the substratebinding pocket [20]. Box D contains the residues 271282 and a conserved arginine at position 275. This box also contains the proposed general-base lysine 274, which forms the S1 substrate-binding pocket in other species [20]. Box E contains residues 304-314, which includes the highly conserved glycine 304, aspartic acid 305, and asparagine 306 as well as aspartic acid 312 and arginine 


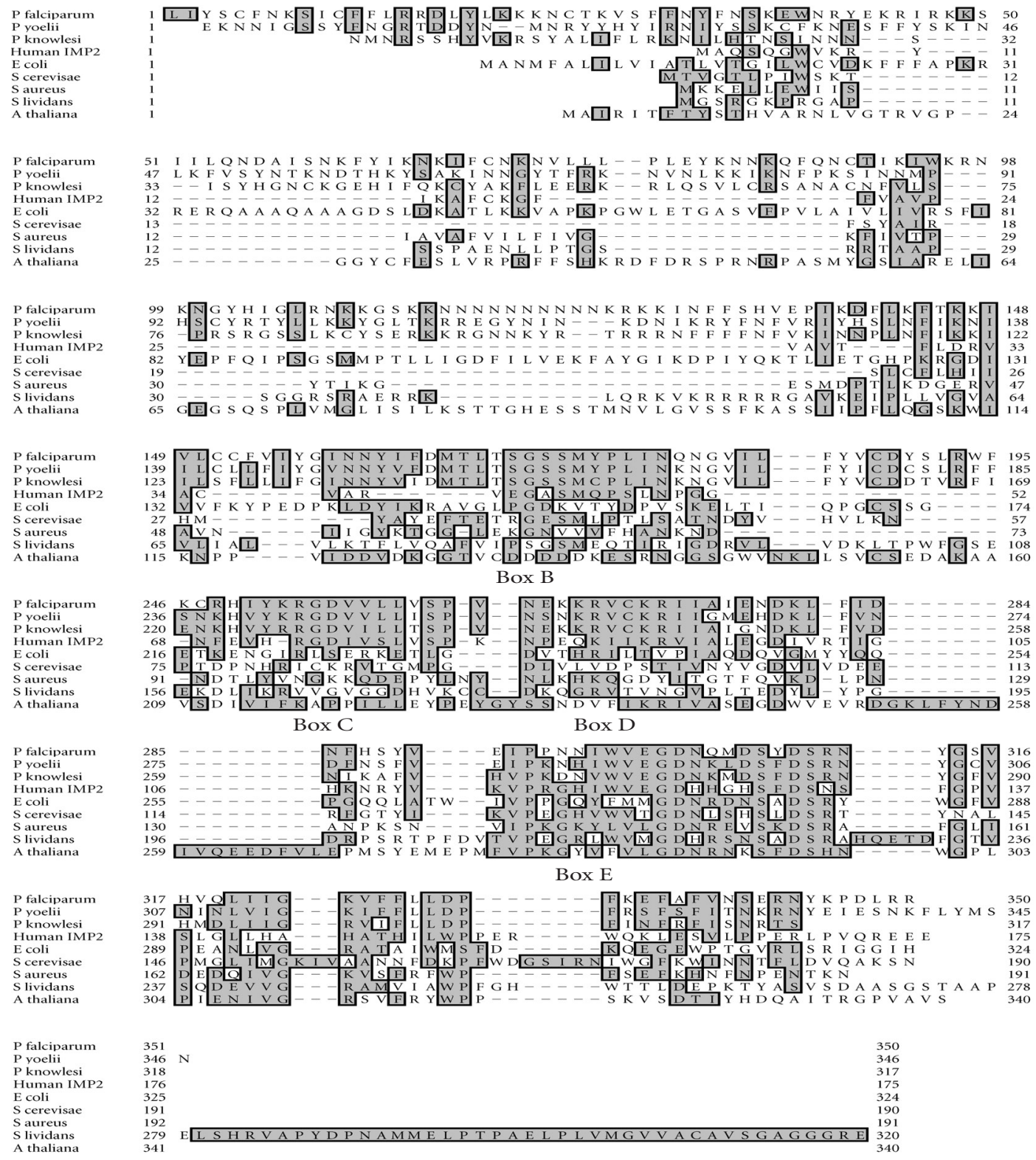

(a)

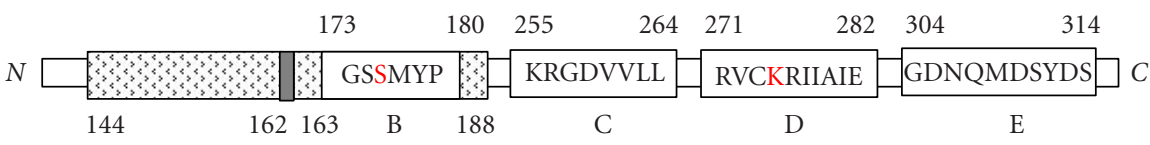

(b)

Figure 2. (a) Multiple alignment of type I signal peptidases from $P$ falciparum (DDBJ/EMBL/GenBank accession number AY582351; protein id AAS91735), $P$ yoelii (DDBJ/EMBL/GenBank accession number AY787663; protein id AAV71057), P knowlesi (DDBJ/EMBL/GenBank accession number AY787662; protein id AAV71056), human IMP2 (mitochondrial inner membrane peptidase NP_115938), E coli (BAA10915), Saccharomyces cerevisae (NP_013870), Staphylococcus aureus (NP_371489), Streptomyces lividans (CAB06808), and Arabidopsis thaliana (CAA71502). The conserved boxes are also labeled. (b) Schematic diagram showing the various conserved boxes B-E and the probable transmembrane regions of type I signal peptidase from $P$ falciparum. Open boxes represent the conserved type I signal peptidase motifs and the amino acid sequence of each motif is written by the single letter code inside the box. Labels below the open boxes (B-E) are the names assigned to the motifs. The amino acids of the catalytic dyad are shown in red. The numbers above the boxes represent the boundaries for each box in $P$ falciparum. Shaded boxes represent the two transmembrane regions and the numbers below the boxes represent the boundaries for these regions. The gray shaded area is the overlapping region in the two transmembrane regions. $N$ and $C$ denote the $N$ and $C$-terminal ends of the protein. 
314. From these data, it is evident that $P$ falciparum type I signal peptidase contains all the conserved domains, which contribute to the catalytic site. It is clear from Figure $2 \mathrm{a}$ that the distances and position of the essential residues for catalysis are well conserved in these Plasmodium species.

The topology of type I signal peptidases varies significantly and it has been reported that the majority of these enzymes from gram-negative bacteria have multiple transmembrane segments at the $N$-terminus for assembly of the enzyme into the membrane and a carboxyterminal catalytic domain $[1,4]$. On the other hand, type I signal peptidases from gram-positive bacteria (e.g., Bacillus subtilis, Staphylococcus aureus, and Streptococcus pneumoniae) and some gram-negative bacteria (e.g., Bradyrhizobium japonicum, Rhodobacter capsulatus, Rickettsia rickettsii, and Rickettsia typhi) are smaller in size with only a single transmembrane segment at the $N$ terminus for the assembly into the membrane $[1,4,21$, 22]. The topology of type I $P$ falciparum signal peptidase can be compared to the signal peptidases of gramnegative bacteria. The secondary structure for the $P$ falciparum type I signal peptidase was examined by using the TMpred program (http://www.ch.embnet.org/ cgi-bin/TMPRED_form_parser). This program predicts two possible models for the transmembrane regions in $P$ falciparum type I signal peptidase. The strongly preferred model with higher score predicted two transmembrane regions (shown in Figure $2 \mathrm{~b}$ ) while the alternative model with lower score predicted only one transmembrane region.

It has been reported previously that in vitro selfcleavage takes place in all the investigated bacterial type I signal peptidases including enzymes from $E$ coli, Bacillus subtilis, and S. pneumoniae [23, 24, 25]. The effect of phospholipids on in vitro self cleavage of $S$ pneumoniae type I signal peptidase has also been studied and it has been shown that in the presence of phospholipids, the self-cleavage occurred at one cleavage site between Gly36-His37 [25]. For biochemical characterization of $P$ falciparum type I signal peptidase, the gene was cloned at BamHI and HindIII sites in the bacterial expression vector pET28b (Novagen), which contains hexa-histidine tag at both the $N$ - and $C$-termini. The resultant construct was transformed into E coli strain BL21(DE3)pLysS. This strain of bacteria lacks an outer membrane protease, which improves recovery of intact recombinant proteins. The transformed $E$ coli cells were grown and induced with IPTG for protein expression at $37^{\circ} \mathrm{C}$ for various times from $30 \mathrm{~min}$ to $4 \mathrm{~h}$. The protein contains 44 additional amino acids of the vector, which includes the histidines at both the $\mathrm{N}$ - and $\mathrm{C}$-terminals. Preliminary studies about localization of the recombinant type I signal peptidase from $P$ falciparum revealed that the protein is localized in the inclusion bodies. Therefore, the bacterial pellet was resuspened in the urea buffer and sonicated to recover the proteins. This lysate was further purified using $\mathrm{Ni}^{2+}$-NTA-agarose matrix. The proteins bound to the $\mathrm{Ni}^{2+}$-NTA-agarose matrix were checked for purity by SDS-PAGE and western blot analysis using anti-His antibodies. Figure $3 \mathrm{a}$ shows the Coomassie blue stained gel and the arrows marked a, b, and c show the possible degraded products of type I signal peptidase (Figure 3a, lane 1). The western blot analysis also detected the same three bands of $\sim 23.6, \sim 22.7$, and $\sim 10.3 \mathrm{kd}$ (Figure 3a, lane 3 ). The appearance of degraded products increased with increase in the time of induction (data not shown). These data show that the preparation does not contain any intact type I signal peptidase protein. The size of the bands in western blots suggests that the type I signal peptidase of $P$ falciparum is degrading autocatalytically at its own active site Gly-SerSer-Met (GSSM) and probably one additional recognition site Ala-Ile-Ser-Asn (AISN) in the $N$-terminal of the protein. The two most likely sites of predicted signal peptidase cleavage and the predicted sizes of the bands after this degradation are shown in Figure 3b. Previous studies of sequences surrounding the cleavage site recognized by type I signal peptidase have shown that Ser can occur at -1 position instead of customary Ala and Gly can occur instead of Ala at -3 position $[14,24]$. The degradation position between residues Ser-59 and Asn-60 conforms to this and the cleavage between Ser-175 and Met176 residues is predicted by the occurrence of Ser-175 and Gly-173 at -1 and -3 positions, respectively. Previous studies have shown that E coli SPase I is also cleaved autocatalytically at a consensus cleavage site at its $\mathrm{N}$-terminus [23].

For studying if the signal peptidase from $P$ falciparum is functionally active, E coli IT41 cells were used. These cells have a single mutant copy of the leader peptidase $\mathrm{B}$ gene and are temperature sensitive for preprotein processing [5]. The strain shows normal growth at $30^{\circ} \mathrm{C}$, but growth is dramatically affected at the nonpermissive temperature $\left(42^{\circ} \mathrm{C}\right)$. This temperature sensitive mutation can be complemented by a plasmid, which carries a functional leader peptidase B gene. In a number of previous studies, this assay has been used to demonstrate type I signal peptidase activity from a variety of sources. These include the enzymes from Salmonella typhimurium [26], Bradyrhizobium japonicum [21, 27], Staphylococcus aureus [28], Streptococcus pneumoniae [29], Streptomyces lividans [30], Bacillus amyloliquefaciens [31], Rickettsia rickettsii and Rickettsia typhi [22], and Legionella pneumophila [16].

The $P$ falciparum type I signal peptidase gene cloned in $\mathrm{pET}-28 \mathrm{~b}$ plasmid was used to evaluate the effect of overexpression of $P$ falciparum signal peptidase on its complementation capacity. Growth of E coli IT41 harboring signal peptidase pET-28b-SP and the respective control plasmid pET-28b was monitored at $42^{\circ} \mathrm{C}$. The results show that type I signal peptidase of $P$ falciparum supported growth at the nonpermissive temperature and it can process all the $E$ coli proteins which are necessary for 


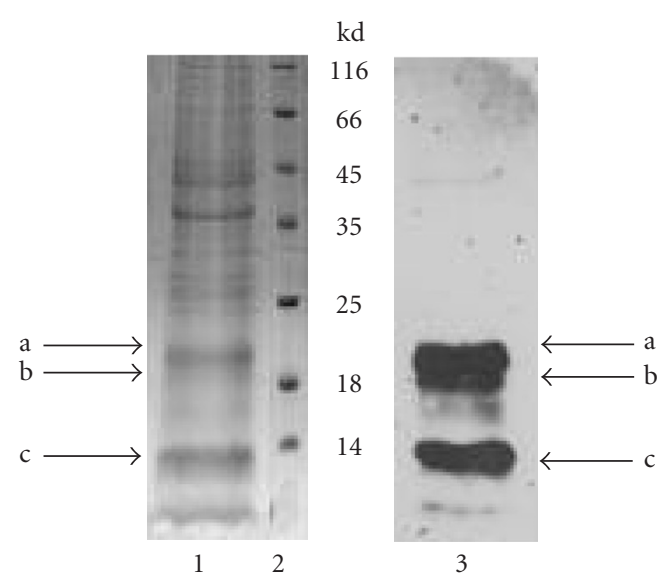

(a)

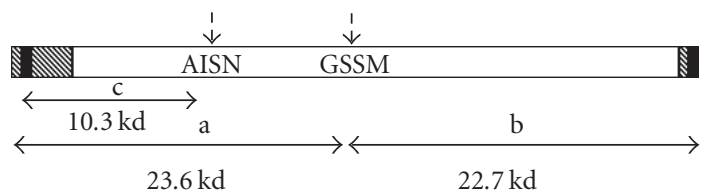

(b)

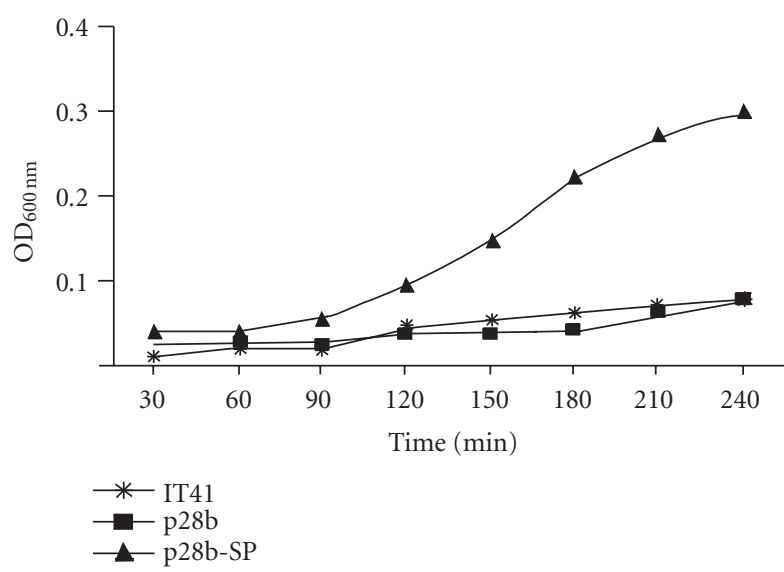

(c)

FIGURE 3. (a) SDS-PAGE and Western blot analysis of recombinant type I signal peptidase from $P$ falciparum. The proteins bound to $\mathrm{Ni}^{2+}$-NTA-agarose matrix were separated by SDS-PAGE and stained with Coomassie brilliant blue. Lane 1 shows the various bands after this staining. The letters a, b, and c correspond to the predicted fragments in panel (b). Lane 2 is the molecular weight marker. The size of molecular weight marker is written on the right side of lane 2 . The same proteins were separated by SDS-PAGE and transferred to nitrocellulose membrane using standard protocols. The blot was probed with His-tag monoclonal antibodies and developed as described in materials and methods. Lane 3 shows that the same bands a, b, and c are reacting with the anti-His antibodies. (b) Size of the predicted degradation products of type I signal peptidase of $P$ falciparum cloned in pET28b. The clear area in the bar demonstrates the sequence of type I signal peptidase, area with vertical bars represents the amino acids of the vectors, and shaded area at the two ends is the His tag. The probable cleavage sites are shown by single-letter code in the box and the dashed vertical arrows indicate the site of cleavage. The horizontal arrows under the bar denote the size and location of the possible products $\mathrm{a}$, b, and $\mathrm{c}$ shown in panel (a). (c) Growth curves of E coli IT41 transformants containing the appropriate plasmids. The cultures grown overnight at $30^{\circ} \mathrm{C}$ were diluted and incubated at $42^{\circ} \mathrm{C}$; the $\mathrm{OD}_{600}$ was monitored as a function of time. All points are the mean of at least two independent experiments.

cell viability (Figure 3c). These data confirm that type I signal peptidase from $P$ falciparum is functionally active.

In this study, we present the cloning and sequence analysis of the putative type I signal peptidase genes from $P$ falciparum, $P$ knowlesi, and $P$ yoelii. The amino acid residues serine and lysine critical for catalytic activity of type I signal peptidase in other species and the catalytic domains (boxes B through E) are shown to be conserved in these parasite species. We have also shown that type I signal peptidase from $P$ falciparum is an intron-less and single-copy gene. The expression and characterization of type I signal peptidase from $P$ falciparum shows that it is degrading autocatalytically at two sites and the complementation assay shows that it is functionally active. Further studies towards identifying the exact cleavage site and production of mutated proteins are in progress. This study is the first step towards the elucidation of one of the important components of the secretory pathway in $P$ falciparum, and should make an important contribution in understanding the complex process of protein targeting in the parasite. 


\section{ACKNOWLEDGMENTS}

The authors are grateful to Dr C. Chitnis (ICGEB, New Delhi) and Dr A. Mohmmed (ICGEB, New Delhi) for providing the genomic DNA of $P$ knowlesi and $P$ yoelii, respectively. We gratefully acknowledge the gift of E coli strain IT41 from Toshifumi Inada, Nagoya University, Japan. The authors also thank the anonymous reviewers for helpful suggestions. This work was supported by Department of Biotechnology Grant (to R. Tuteja). Infrastructural support from the Department of Biotechnology, Government of India, is gratefully acknowledged. The sequences reported in this paper have been submitted to the GenBank and the accession numbers are PfSPB AY582351, PkSP AY787662, and PySP AY787663.

\section{REFERENCES}

[1] Paetzel M, Dalbey RE, Strynadka NC. The structure and mechanism of bacterial type I signal peptidases. A novel antibiotic target. Pharmacol Ther. 2000;87:27-49.

[2] Paetzel M, Karla A, Strynadka NC, Dalbey RE. Signal peptidases. Chem Rev. 2002;102(12):4549-4580.

[3] Date T. Demonstration by a novel genetic technique that leader peptidase is an essential enzyme of Escherichia coli. J Bacteriol. 1983;154(1):76-83.

[4] Klug G, Jager A, Heck C, Rauhut R. Identification, sequence analysis, and expression of the lepB gene for a leader peptidase in Rhodobacter capsulatus. Mol Gen Genet. 1997;253(6):666-673.

[5] Inada T, Court DL, Ito K, Nakamura Y. Conditionally lethal amber mutations in the leader peptidase gene of Escherichia coli. J Bacteriol. 1989;171(1):585587.

[6] Bron S, Bolhuis A, Tjalsma H, Holsappel S, Venema G, van Dijl JM. Protein secretion and possible roles for multiple signal peptidases for precursor processing in bacilli. J Biotechnol. 1998;64(1):3-13.

[7] Tjalsma H, Noback MA, Bron S, Venema G, Yamane K, van Dijl JM. Bacillus subtilis contains four closely related type I signal peptidases with overlapping substrate specificities. Constitutive and temporally controlled expression of different sip genes. $J$ Biol Chem. 1997;272(41):25983-25992.

[8] Tjalsma H, van den Dolder J, Meijer WJ, Venema G, Bron S, van Dijl JM. The plasmid-encoded signal peptidase SipP can functionally replace the major signal peptidases SipS and SipT of Bacillus subtilis. J Bacteriol. 1999;181(8):2448-2454.

[9] Dalbey RE, Lively MO, Bron S, van Dijl JM. The chemistry and enzymology of the type I signal peptidases. Protein Sci. 1997;6(6):1129-1138.

[10] Bannister LH, Hopkins JM, Fowler RE, Krishna S, Mitchell GH. A brief illustrated guide to the ultrastructure of Plasmodium falciparum asexual blood stages. Parasitol Today. 2000;16(10):427-433.
[11] Nacer A, Berry L, Slomianny C, Mattei D. Plasmodium falciparum signal sequences: simply sequences or special signals?. Int J Parasitol. 2001;31(12):13711379.

[12] Waller RF, Reed MB, Cowman AF, McFadden GI. Protein trafficking to the plastid of Plasmodium falciparum is via the secretory pathway. EMBO J. 2000;19(8):1794-1802.

[13] Wu Y, Wang X, Liu X, Wang Y. Data-mining approaches reveal hidden families of proteases in the genome of malaria parasite. Genome Res. 2003;13(4): 601-616.

[14] von Heijne G. Patterns of amino acids near signalsequence cleavage sites. Eur J Biochem. 1983;133(1): $17-21$.

[15] Rafati S, Salmanian AH, Taheri T et al. Type I signal peptidase from Leishmania is a target of the immune response in human cutaneous and visceral leishmaniasis. Mol Biochem Parasitol. 2004;135(1):13-20.

[16] Lammertyn E, Van Mellaert L, Meyen E et al. Molecular and functional characterization of type I signal peptidase from Legionella pneumophila. Microbiology. 2004;150(Pt 5):1475-1483.

[17] Trager W, Jensen JB. Human malaria parasites in continuous culture. Science. 1976;193(4254):673675.

[18] Sambrook J, Fritsch EF, Maniatis T, eds. Molecular Cloning: A Laboratory Manual. 2nd edition. New York, NY:Cold Spring Harbor Laboratory Press; 1989.

[19] Harlow E, Lane D. Antibodies: A Laboratory Manual, New York, NY:Cold Spring Harbor Laboratory Press:1988.

[20] van Roosmalen ML, Jongbloed JD, Dubois JY, Venema G, Bron S, van Dijl JM. Distinction between major and minor Bacillus signal peptidases based on phylogenetic and structural criteria J Biol Chem. 2001;276(27):25230-25235.

[21] Bairl A, Muller P. A second gene for type I signal peptidase in Bradyrhizobium japonicum, sipF, is located near genes involved in RNA processing and cell division. Mol Gen Genet. 1998;260(4):346-356.

[22] Rahman MS, Simser JA, Macaluso KR, Azad AF. Molecular and functional analysis of the lepB gene, encoding a type I signal peptidase from Rickettsia rickettsii and Rickettsia typhi. J Bacteriol. 2003;185(15):4578-4584.

[23] Talarico TL, Dev IK, Bassford PJ Jr, Ray PH. Intermolecular degradation of signal peptidase I in vitro. Biochem Biophys Res Commun. 1991;181(2):650656.

[24] van Roosmalen ML, Jongbloed JD, Kuipers A, Venema G, Bron S, van DijL JM. A truncated soluble Bacillus signal peptidase produced in Escherichia coli is subject to self-cleavage at its active site. J Bacteriol. 2000;182(20):5765-5770. 
[25] Peng SB, Wang L, Moomaw J et al. Biochemical characterization of signal peptidase I from grampositive Streptococcus pneumoniae. J Bacteriol. 2001; 183(2):621-627.

[26] van Dijl JM, van den Bergh R, Reversma T, Smith H, Bron S, Venema G. Molecular cloning of the Salmonella typhimurium lep gene in Escherichia coli. Mol Gen Genet. 1990; 223(2):233-240.

[27] Muller P, Ahrens K, Keller T, Klaucke A. A TnphoA insertion within the Bradyrhizobium japonicum sipS gene, homologous to prokaryotic signal peptidases, results in extensive changes in the expression of PBM-specific nodulins of infected soybean (Glycine max) cells. Mol Microbiol. 1995;18(5):831-840.

[28] Cregg KM, Wilding I, Black MT. Molecular cloning and expression of the spsB gene encoding an essential type I signal peptidase from Staphylococcus aureus. J Bacteriol. 1996;178(19):5712-5718.

[29] Zhang YB, Greenberg B, Lacks SA. Analysis of a Streptococcus pneumoniae gene encoding signal peptidase I and overproduction of the enzyme. Gene. 1997;194(2):249-255.

[30] Parro V, Schacht S, Anne J, Mellado RP. Four genes encoding different type I signal peptidases are organized in a cluster in Streptomyces lividans TK21. Microbiology. 1999;145(Pt 9):2255-2263.

[31] Chu HH, Hoang V, Kreutzmann P, Hofemeister B, Melzer M, Hofemeister J. Identification and properties of type I-signal peptidases of Bacillus amyloliquefaciens. Eur J Biochem. 2002;269(2):458-469. 

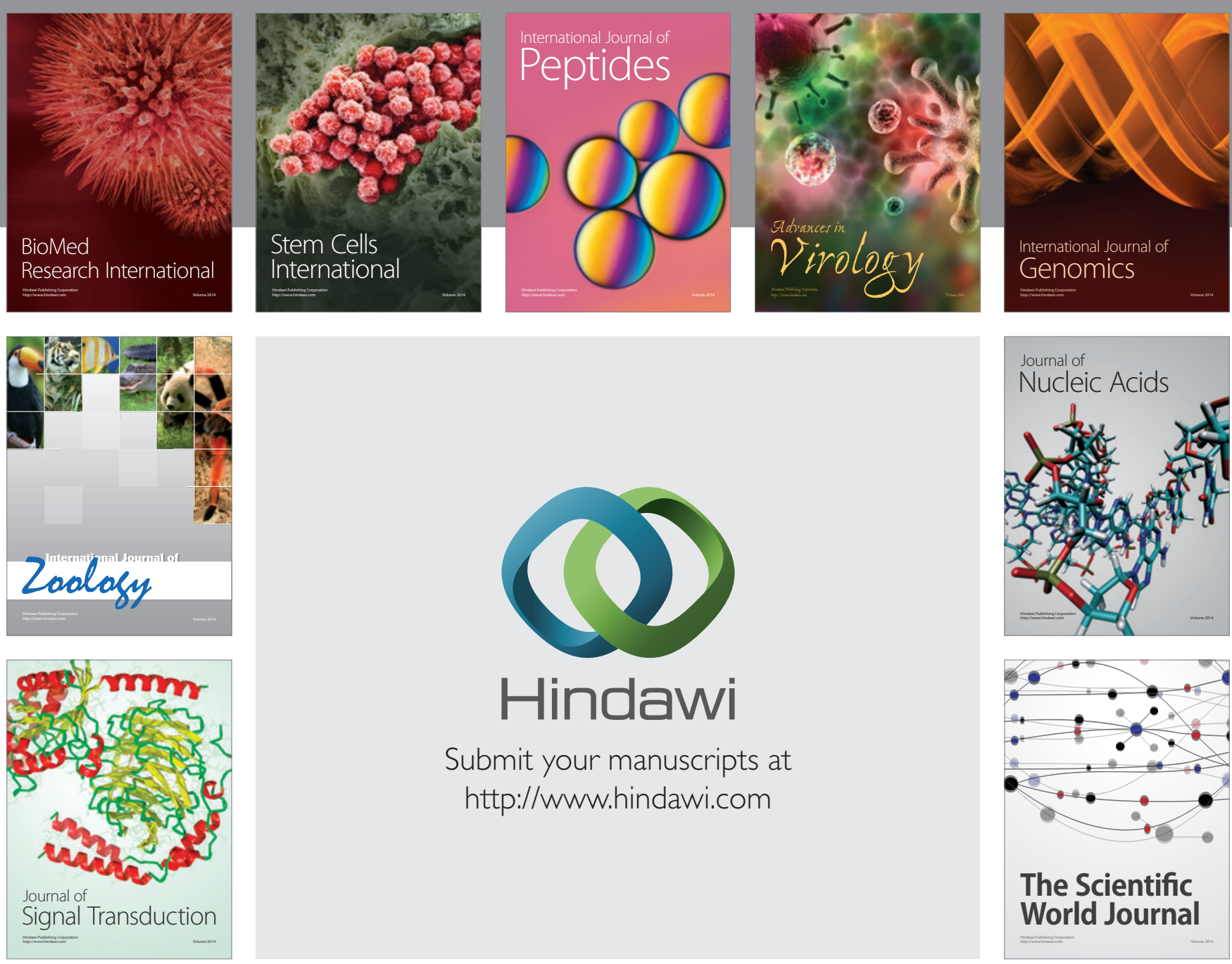

Submit your manuscripts at

http://www.hindawi.com
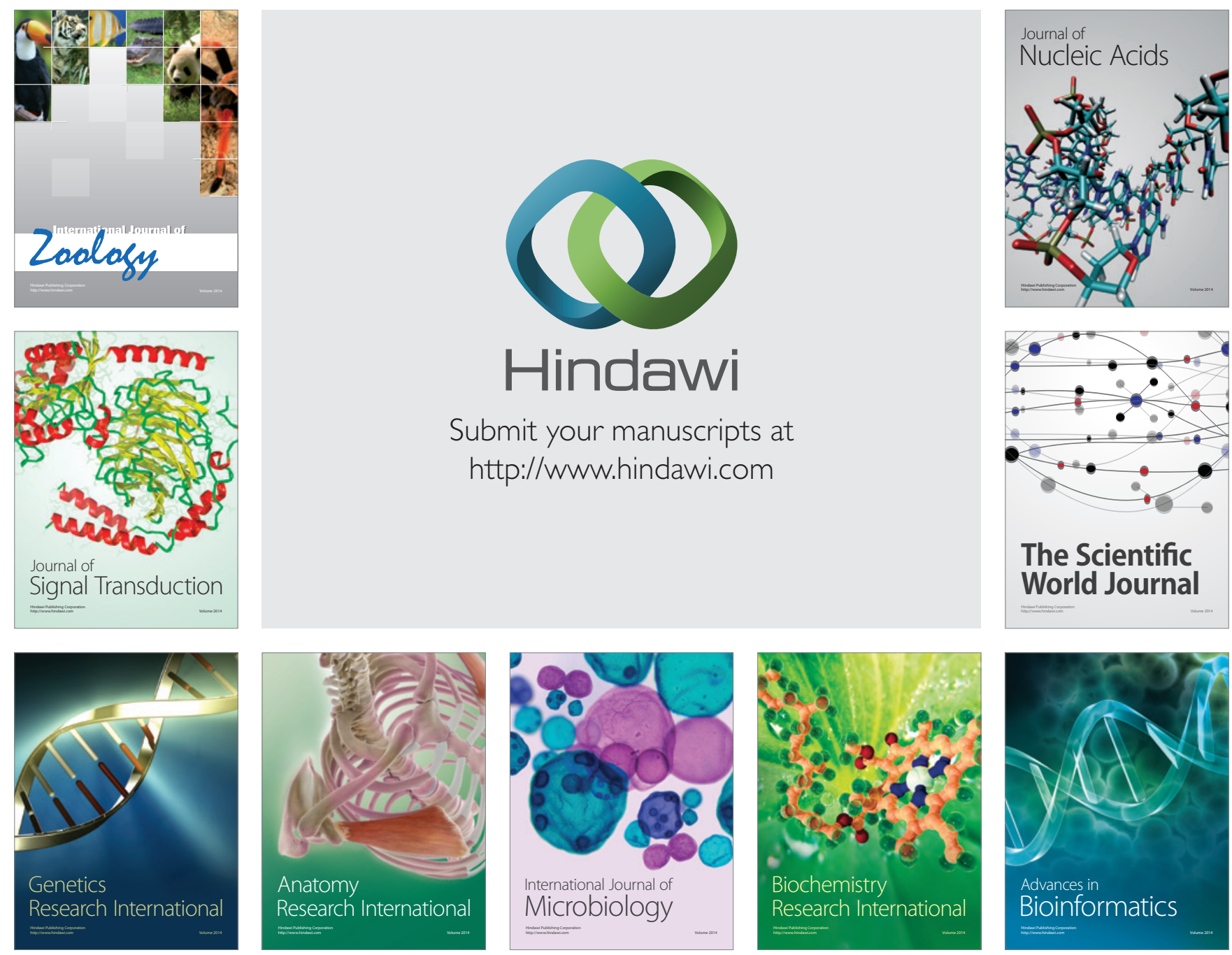

The Scientific World Journal
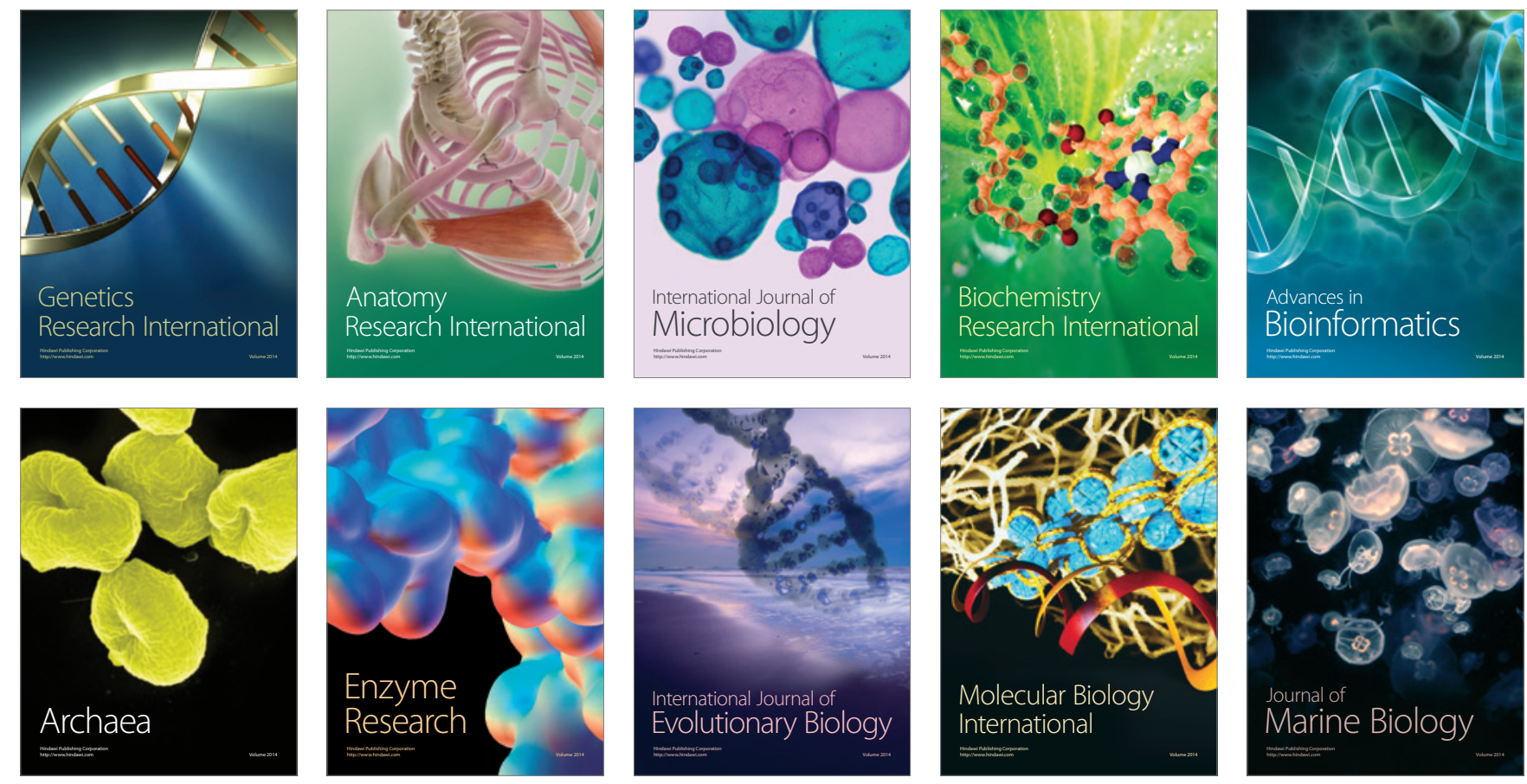